\title{
An Adaptive Target Extraction Algorithm Based on Energy Functional
}

\author{
Wei Chuan $\mathrm{Ni}^{1, a^{*}}$, Wei Jian $\mathrm{Mo}^{2, \mathrm{~b}}$ \\ ${ }^{1}$ Xinhua College of Sun Yat-sen University, Guangzhou 510520,China) \\ ${ }^{2}$ Xinhua College of Sun Yat-sen University, Guangzhou 510520,China) \\ a544737200@qq.com, ${ }^{\mathrm{b}} 472252720 @ q q . c o m$
}

Keywords: Segmentation algorithm; Energy functional; Probability plots; Profile; Target.

\begin{abstract}
Objective: Solve the problem that was the low extract accuracy of existing target extraction algorithm. Methods: Proposes an adaptive object extraction algorithm based on energy functional. Article algorithm was defined the average item and the contour curve for the energy functional, which combining with the probability graph and differential geometry. At final, we would be getting the adaptive energy functional. Results: Article algorithm can effectively extract the target contour. And it is not affected by the background signal. Conclusion: From the contour detection experiments and algorithms comparative experiments, we can see that the algorithm can accurately extract the desired signal. Simultaneously, it can maintain a higher visual for the target.
\end{abstract}

\section{Introduction}

Nowadays, Target extraction algorithm has an unprecedented space for development. Target extraction algorithm can effectively extract the signal, because it was according to certain rules. So it was plays a vital role in the image processing and analyze. Nowadays, traditional target extraction algorithm has many algorithms [1-4]. For example, the target extraction algorithm based on threshold[5], target extraction algorithm based on histogram[6], and Target extraction algorithm based clustering method[7], etc. In recent years, researchers propose a new target extraction algorithm that based on energy functional. It can effectively improve the extraction effect of target, because it was good at using the image characteristics to analysis and processing. But it still exist the problems that susceptible to distortion and interference.

In response to these problems, Article algorithm was using probability plots and means algorithm to analyze the target image. And Article algorithm was using that to optimization the energy functional. It was optimizing the energy functional in terms of the mean and outline to improve the accuracy of object extraction. Make it can always kept higher target resolution in different background environment.

\section{Energy functional}

Hypothesis $\mathrm{u}(x, y): \Omega \subset R^{N} \rightarrow R(N=2,3)$ as a function of the image field can be adjusted, Bounded open set is $\Omega$, The original image is $\mathrm{u}_{0}(x, y)$, Without loss of generality, Hypothesis $0 \leq \mathrm{u}_{0}(x, y) \leq 1,(x, y) \subset \Omega$, and Looking for $(u, C)$ make the following energy functional minimum[8-9]. Firstly, we can definition of a non-negative constant $\alpha$ and $\beta$.

$$
\text { So } F(u, C)=\iint_{\Omega}\left|u(x, y)-u_{0}(x, y)\right|^{2} d x d y+\alpha \iint_{\Omega \backslash C}|\nabla u(x, y)|^{2} d x d y+\beta \int_{C} d \sigma .
$$


The first formula was the fidelity term. $\iint_{\Omega}\left|u(x, y)-u_{0}(x, y)\right|^{2} d x d y$. It was used to control similarity of the extracted image. The second formula was regularization term. $\iint_{\Omega \backslash C}|\nabla u(x, y)|^{2} d x d y$. It was used to ensure the smoothness of the extracted image. The third formula was length items of curved contour C. $\int_{C} d \sigma$. It was used to control the roughness of the edge.

First of all, Energy functional was using the piecewise constant to approximate the various parts of the image. And researchers was combined the variational method to improvement the energy functional. So we can give it like the following expression.

$$
F\left(C, c_{1}, c_{2}\right)=\lambda_{1} \iint_{\Omega i n}\left|u(x, y)-c_{1}\right|^{2} d x d y+\lambda_{2} \iint_{\Omega o u t}\left|u(x, y)-c_{2}\right|^{2} d x d y+\eta|C|
$$

$c_{1}$ and $c_{2}$ was the background mean and objectives mean of both sides of the curved profile C.

And $\eta 、 \lambda_{1} 、 \lambda_{2}$ was the constant. Afterward, it was using the Level set function $\varphi: \Omega \rightarrow R$ represents the evolution curve C. Command that $C=\{(x, y) \in \Omega: \varphi(x, y)=0\}$, definition $\varphi$ was coincided in the Interior region of C, and $\varphi$ was Negative outside of C. Using it to detect the unknown curve C. The new energy functional was like the following expression.

$$
F\left(\varphi, c_{1}, c_{2}\right)=\lambda_{1} \iint_{\varphi \gg 0}\left|u(x, y)-c_{1}\right|^{2} d x d y+\lambda_{2} \iint_{\varphi(0}\left|u(x, y)-c_{2}\right|^{2} d x d y+\eta|C|(\varphi=0)
$$

In the end, we can combine with the Heaviside Function $H(x)$ and the Dirac Function $\delta(x)$ to Improve the following expression like the following expression.

$$
\left.F\left(\varphi, c_{1}, c_{2}\right)=\lambda_{1} \iint_{\varphi \geq 0}\left|u(x, y)-c_{1}\right|^{2} H(\varphi(x, y)) d x d y+\lambda_{2} \iint_{\varphi \ll 0}\left|u(x, y)-c_{2}\right|^{2}(1-H(\varphi(x, y))) d x d y\right)+\eta|C|
$$

\section{Article algorithm}

Probability analysis. Probability plots by using graph theory and probability plots to description diversified effective model. So it was construction of the Probabilistic Graphical Models $G=(V, \varepsilon)$, Therein $V=\{1,2, \ldots, \mathrm{N}\}$ is used to represent the set of nodes. $\varepsilon \subset V \times V$ is denotes an set on top of the image[10].
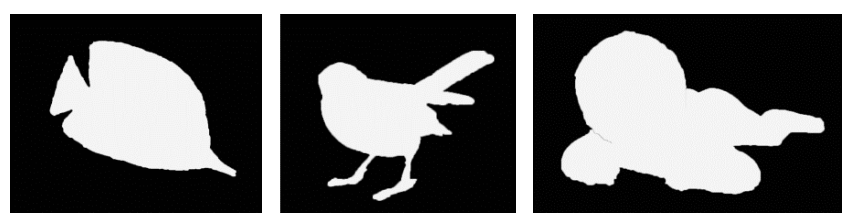

Figure. 1 Image probability map

Hypothesis each node meet that $s \in V$. And $X_{s}$ was the Variable, which denoted as $X_{s}=\{0,1, \cdots, m-1\}$. In order to limit the random Value, make it satisfies the following two properties. (1) $\pi(\omega)>0$ (2) $P\left(X_{s}=x \mid X_{0}, X_{1}, \cdots, X_{n}\right)=P\left(X_{n+1}=X \mid X_{n}\right)$. According to the distribution system of neighborhood $\delta(s), \quad P\left(X_{s} \mid X_{r}, r \in \delta(s)\right)=Z^{-1} \times e^{-\frac{1}{T} \sum_{c \in C} V_{c}\left(X_{S} \backslash X_{r}\right)}$. Therein $\mathrm{Z}$ is 
normalization constant or partition function, $\mathrm{T}$ is energy function. So we can get the following probability map.

In order to extract a reasonable target signal, article algorithm was further analysis of the probability map. Method is as follows. Hypothesis $\omega_{1}$ is the background pixels, and $\omega_{2}$ is the target pixel. We can the probability of $P\left(\mathrm{f} / \omega_{1}\right)$ 和 $P\left(\mathrm{f} / \omega_{2}\right)$ were the density probability of background and objective pixels. Through the use a Gaussian functions to calculate the density probability function. The expression as follows:

$$
P\left(\mathrm{f} / \omega_{1}\right)=\frac{1}{\mathrm{n} \times \mathrm{m}} \sum_{i, j=1}^{\mathrm{n} \times \mathrm{m}} \frac{1}{\sqrt{2 \pi \sigma_{1}^{2}}} e^{-\frac{\left(f-g_{i, j}\right)^{2}}{2 \sigma_{1}^{2}}} ; \quad P\left(\mathrm{f} / \omega_{2}\right)=\frac{1}{\mathrm{n} \times \mathrm{m}} \sum_{i, j=1}^{\mathrm{n} \times \mathrm{m}} \frac{1}{\sqrt{2 \pi \sigma_{2}^{2}}} e^{-\frac{\left(f-k_{i, j}\right)^{2}}{2 \sigma_{2}^{2}}}
$$

There in $\sigma_{1} 、 \sigma_{2}$ respectively is a function of the density of the background and target, $\mathrm{f}$ is the total value of the image pixels, $g_{i, j} 、 k_{i, j}$ are pixel in background and objectives, and $\mathrm{n} \times \mathrm{m}$ is the current size of the image.

In the end, we can obtain a high accuracy background mean and target mea. As follows:

$$
\text { Background Mean: } A\left(P\left(\mathrm{f} / \omega_{1}\right)\right)=\frac{P\left(\mathrm{f} / \omega_{1}\right) \cdot f}{n \times m} \quad \text { (3) Target Mean: } A\left(P\left(\mathrm{f} / \omega_{2}\right)\right)=\frac{P\left(\mathrm{f} / \omega_{1}\right) \cdot f}{n \times m}
$$

Simplified the Length entry. According to the definition of differential geometry, Profile curve is defined as $\int_{L} n_{L} d L=2|C|$.

Therein $|C|$ is the Euclidean distance length of the contour curve C. $n_{L}$ is the measure in a straight line $\mathrm{L}$ and contour C compared to the straight set. Therefore, Set up $1 \quad(1 \geq 0)$ is distance between the straight line $\mathrm{L}$ to the coordinate origin $\mathrm{O}$. $\theta \quad(0 \leq \theta \leq 2 \pi)$ is the angle between the $\mathrm{x}$ axis and the straight line L. So: $\zeta=\left\{(1, \theta) \in R^{2} ; 1 \geq 0,0 \leq \theta \leq 2 \pi\right\}$,

Set up $d L=d l d \theta$, using it Substituting into equation (5): $\iint_{L} n_{L} d L=\iint_{L} n_{L}(1, \theta) d l d \theta=2|C|$

We can get the length term for profile curve: $|C|=\frac{1}{2} \sum_{k} n_{k} \frac{\delta^{2} \Delta \theta_{k}}{\left|e_{k}\right|}$, Therein $\delta$ is noise value Set up $\omega_{k}=\frac{\delta^{2} \Delta \theta_{k}}{\left|e_{k}\right|}$, Can be simplified to: $|C|=\frac{1}{2} \sum_{k} n_{k} \omega_{k}$
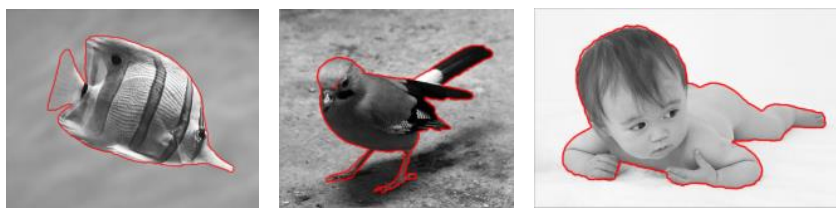

Figure. 2 Profile curve of the image

In summary, According to mean equation (3)、(4) and Contour curve length items equation (5), substituted into the energy functional (2). Finally obtain the following expression.

$$
\left.F\left(\varphi, c_{1}, c_{2}\right)=\lambda_{1} \iint_{\varphi \geq 0}\left|u(x, y)-A\left(P\left(\mathrm{f} / \omega_{1}\right)\right)\right|^{2} H(\varphi) d x d y+\lambda_{2} \iint_{\varphi l 0}\left|u(x, y)-A\left(P\left(\mathrm{f} / \omega_{2}\right)\right)\right|^{2}(1-H(\varphi)) d x d y\right)
$$




\section{Simulation experiments}

In order to verify the effectiveness of the proposed method, using the simulation environment is MATLAB 2014a.

Process of Contour Detection. Checking algorithm was selection of the different types of images. Therein Figure 3. (a) and (b)were simple background image, and Figure 3. (c) and (b) were complex background image.
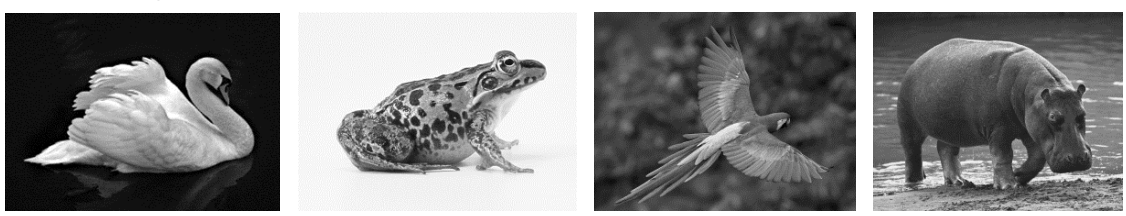

Figure. 3 Original image to be processed

Step1: It was Random search initialization contour.

Step2: Further search outline of the image based on probability plots.

Step3: Eventually extracted the contour signal of the image.
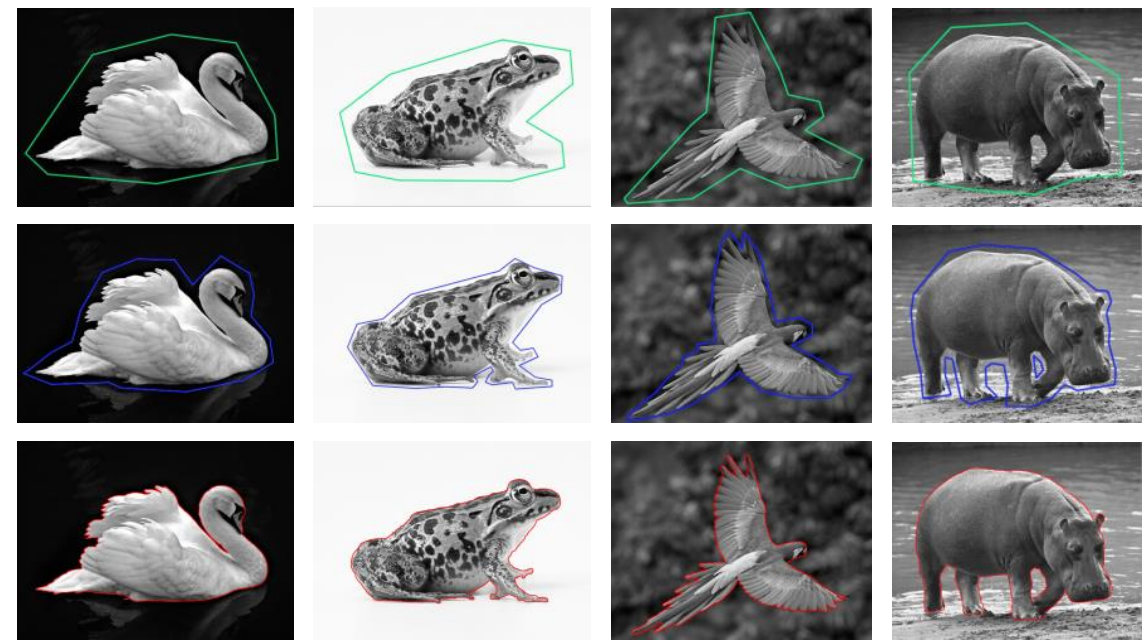

Figure. 4 Contour detection map of the images

Compared with the traditional algorithm. In order to facilitate observation of the target signal, article algorithm was using the edge detection algorithm to get the target edge signal. And using the processing results compared with the traditional energy functional algorithm.
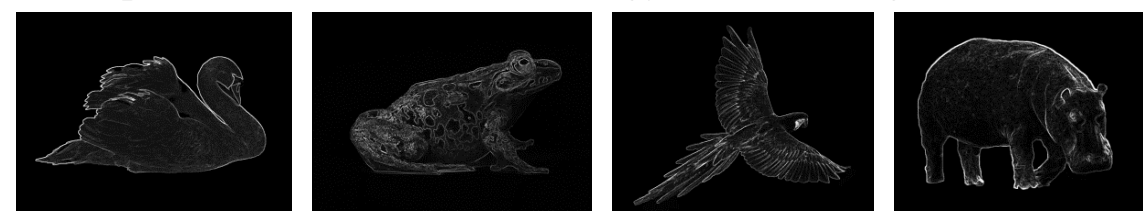

Figure. 5 Each image edge detection effect diagram
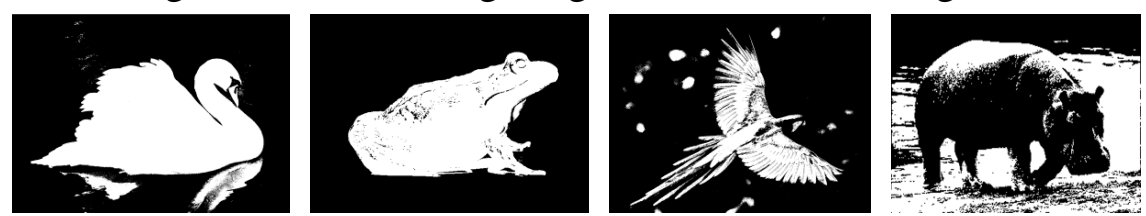

Figure. 6 The traditional energy functional algorithm 

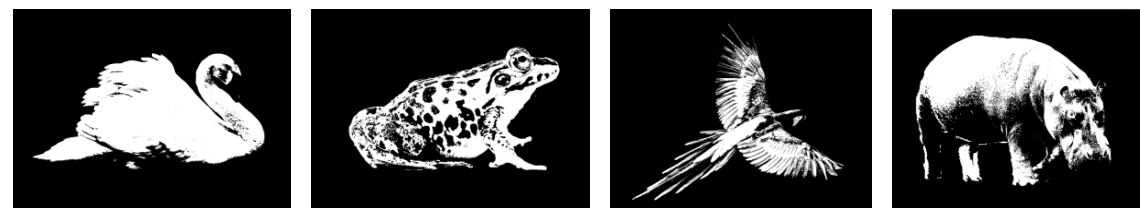

Figure. 7 The effect diagram after using article algorithm

By observing Figures 5 to 7 can be see that the traditional energy functional can effectively extract the target signal, but it was susceptible to interference in the case of complex background. For the extracted target image, Easily find that article algorithm can get the higher target details.

\section{Summary}

Proposed an adaptive target extraction algorithm based on energy functional. Article algorithm was studied by conventional energy functional and redefining it. In order to get a better the extraction effect for the article algorithm. We were optimization of the mean terms and profile curves. Experimental results show that article algorithm can quickly identify the target contour signal from the image. Compared with the traditional target extraction algorithm, prove that article algorithm has higher extraction accuracy.

\section{References}

[1] Chaira Tamalika. A rank ordered filter for medical image edge enhancement and detection using intuitionistic fuzzy set[J].Applied Soft Computing.2012,12(4):1259-1266.

[2] Somkantha Krit, Theera-Umpon Nipon, Auephanwiriyakul Sansanee. Boundary Detection in Medical Images Using Edge Following Algorithm Based on Intensity Gradient and Texture Gradient Features[J]. IEEE Transactions On Biomedical Engineering.2011, 58(3): 567-573.

[3] Han Xian-Hua, Chen Yen-Wei. A robust method based on ICA and mixture sparsity for edge detection in medical images [J]. Signal Image And Video Processing.2011, 5(1): 39-47.

[4] $\mathrm{Du} \mathrm{Ke}$, Peng Lin. New algorithms for preserving edges in low-bit-rate wavelet-based image compression[J]. Wiley-blackwell.2012,7(6): 539-545.

[5] Xiang DL, Tang T, Hu CB, Li Y, Su Y. A Kernel Clustering Algorithm With Fuzzy Factor: Application to SAR Image Segmentation [J]. IEEE GEOSCIENCE AND REMOTE SENSING LETTERS. 2014, 11(7): 1290-1294.

[6] Rahman MM, Horiguchi S. A fully unsupervised color textured image segmentation algorithm using weighted mean histograms features [J]. SIGNAL IMAGE AND VIDEO PROCESSING. 2012,6(02): 197-209.

[7] Osuna-Enciso V, Cuevas E, Sossa H. A comparison of nature inspired algorithms for multi-threshold image segmentation [J]. EXPERT SYSTEMS WITH APPLICATIONS. 2013, 40(04): 1213-1219.

[8] Liu GQ, Zhou ZH, Xie SL. Global minimization of adaptive local image fitting energy for image segmentation [J]. Journal of Systems Engineering and Electronics. 2014,25(2): 307-313.

[9] Zeng XL. Chen DG. Image segmentation algorithm of regional information based on generalization ability[J]. International Journal of Applied Mathematics and Statistics. 2013, 51(24): 265-273.

[10]Avraham T, Lindenbaum M. Esaliency (Extended Saliency): Meaningful Attention Using Stochastic Image Modeling [J]. IEEE TRANSACTIONS ON PATTERN ANALYSIS AND MACHINE INTELLIGENCE. 2010, 32(4): 693-708. 